\title{
IMPACTOS DAS ONDAS DE FRIO SOBRE A SAÚDE PÚBLICA NO MUNICÍPIO DE SÃO JOAQUIM, SANTA CATARINA, BRASIL
}

\author{
SILVEIRA, Rafael Brito - rafael.brito@posgrad.ufsc.br \\ Universidade Federal de Santa Catarina / UFSC \\ MENDONÇA, Magaly + - magaly@cfh.ufsc.br \\ Universidade Federal de Santa Catarina / UFSC \\ FRANKE, Alberto Elvino - alberto.franke@ufsc.br \\ Universidade Federal de Santa Catarina / UFSC
}

\begin{abstract}
BITENCOURT, Daniel Pires - daniel.bitencourt@fundacentro.gov.br
Fundação Jorge Duprat Figueiredo de Segurança e Medicina do Trabalho /

FUNDACENTRO
\end{abstract}

\begin{abstract}
RESUMO: Para várias partes do mundo têm sido conduzidos estudos que tratam dos impactos dos extremos de temperatura sobre a saúde pública. Neste trabalho é apresentada uma análise da ocorrência de ondas de frio (OdF), assim como os impactos destes eventos no número de internações hospitalares do município de São Joaquim, Santa Catarina (SC), Brasil. Por meio de avaliações estatísticas, foram comparados dados de temperatura média do ar e de número de dias com OdF com dados de internações por doenças respiratórias e circulatórias. Observou-se que São Joaquim é um município com significativa ocorrência de OdF, com média de 3,5 OdF/ano. Durante os eventos, as temperaturas são em geral rigorosamente baixas, principalmente no inverno austral. Os resultados comparativos apontam com mais clareza impactos do frio sobre as internações hospitalares devido à pneumonia. O frio no município de São Joaquim explica 39 \% da morbidade por pneumonia. Também foram encontrados indícios de que os maiores impactos na saúde pública local sejam causados por invernos que apresentem número maior de dias frios no decorrer da estação, mesmo que o frio não seja tão rigoroso.
\end{abstract}

PALAVRAS-CHAVES: Ondas de frio, Morbidade, Doenças respiratórias, Doenças circulatórias.

IMPACTS OF COLD WAVES ON PUBLIC HEALTH IN SÃO JOAQUIM, SANTA CATARINA, $B R A Z I L$

ABSTRACT: Studies have been conducted in different parts of the world to address the impacts of extremes temperature on the public health. This paper presents an analysis of the occurrence of cold waves, as well as the impact of these events on the number of hospital admissions in the city of São Joaquim, Santa Catarina (SC), Brazil. Through statistical analysis, it was performed comparisons between the mean temperature and number of cold days with data of the morbidity from circulatory and respiratory diseases. It was observed that São Joaquim is a municipality with significant occurrence of cold wave, with an average of 3.5 waves per year. During the events, the temperatures are generally strictly low, especially in the austral winter. The comparative results showed more clearly the impacts of cold on hospital admissions due to pneumonia. The cold in the municipality of São Joaquim explains 39\% of the morbidity due to pneumonia. Evidence has also been found that the greatest impacts on local public health are caused by winters with more cold days in the season, even if the cold is not too severe.

KEYWORDS: Cold wave, Morbidity, Respiratory diseases, Circulatory diseases. 


\section{INTRODUÇÃO}

A região Sul do Brasil, por localizar-se ao sul do Trópico de Capricórnio, faz parte da porção subtropical do hemisfério sul. Por isso, ocorrem variabilidades climáticas sazonais, com as estações de transição (outono e primavera) podendo apresentar tanto dias mais quentes quanto dias mais frios. No verão, geralmente os dias caracterizam-se por temperaturas mais elevadas e no inverno há dias e noites com temperaturas baixas. Em Santa Catarina (SC) as temperaturas tendem a aumentar no sentido sul-norte e das áreas mais elevadas do planalto para oeste e leste (MONTEIRO; MENDONÇA, 2014). A posição geográfica de SC favorece a atuação de muitos sistemas atmosféricos, com grande amplitude térmica no ciclo anual (GRIMM, 2009). No que diz respeito ao frio, normalmente, quando uma massa de ar frio avança sobre a região Sul do Brasil, ocorrem ventos fortes do quadrante sul e queda significativa da temperatura do ar (MONTEIRO; MENDONÇA, 2014). Nos meses de inverno é bastante comum a atuação de sistemas frontais, os quais são seguidos por massas de ar frio que podem atuar com grande frequência e intensidade (MURARA; FUENTES, 2014).

O município de São Joaquim localiza-se no Planalto Sul catarinense, na Serra Geral, a uma altitude média de 1.360 metros (IBGE, 2010). Por isso, normalmente as condições de frio que ocorrem em maior escala espacial são potencializadas localmente em São Joaquim. Em altitudes como esta, os efeitos da perda radiativa, devido à atuação de massas de ar frio, e de advecção de ar frio, quando o vento sopra predominantemente de sul, favorecem acentuados declínios de temperatura, que frequentemente atingem valores negativos.

Muitos estudos abordam a influência do frio sobre a saúde da população, quando em geral as pessoas apresentam enfermidades associadas aos aparelhos respiratório ou circulatório (e.g. FEIGIN et al., 2000; WILKINSON et al., 2001; HEALY, 2003; WHO, 2004; MORABITO et al., 2006; CARSON et al., 2006; GUERREIRO, 2011; BARNETT et al., 2012; GASPARRINI et al., 2015; FANTE; ARMOND, 2016). No caso de problemas no aparelho respiratório, o agravo das enfermidades é causado por efeitos no epitélio da via aérea, tecido que reveste a mucosa das fossas nasais. Sendo constatado em distintas partes do mundo e afetando grupos diversos, as infecções virais são facilitadas em condições de frio, uma vez que as pessoas buscam permanecer em lugares fechados por mais tempo a fim de se aquecer (BRASIL, 2014). Segundo Guerreiro (2011), as pessoas muito jovens ou idosas geralmente são mais afetadas devido à menor autonomia, ou seja, indivíduos que dependem mais de terceiros e, por conseguinte, apresentam maiores dificuldades para, por exemplo, realizar algum deslocamento necessário, buscar auxílio médico/farmacêutico e fazer uso de medicamentos. O frio é ainda apontado como uma importante causa para o afastamento do trabalho devido às doenças respiratórias (BITENCOURT et al., 2009) e, portanto, é também um problema à saúde ocupacional.

Em relação às doenças do aparelho circulatório, as mudanças dos tipos de tempo influenciam a temperatura corporal, que por sua vez acionam os mecanismos homeostáticos humanos para o controle do equilíbrio entre 0 ambiente externo e o organismo interno. Como forma de defesa o organismo responde a este processo ativando o funcionamento da vasoconstrição e vasodilatação, aumentando ou diminuindo o fluxo dos vasos sanguíneos, 
podendo desencadear problemas de saúde (TORTORA, 2000). As baixas temperaturas causam contração das artérias para ajudar o corpo humano a reter o calor; devido ao estreitamento, a existência de possíveis placas de gordura e coágulos, pode ocorrer o bloqueio do fluxo sanguíneo para o coração. Por mais que esse comportamento seja um mecanismo natural de assistência contra o frio, a vasoconstrição pode aumentar o risco de infarto, AVC e outras doenças isquêmicas do coração (FM/UFMG, 2014).

Assumindo a hipótese de que nas porções mais altas da região Sul o frio apresenta impactos maiores à saúde pública do que nas porções de menor altitude, este artigo objetiva mostrar através de metodologia própria qual é a influência das ondas de frio (OdF) na saúde da população de São Joaquim, através da comparação dos dados de temperatura do ar com dados de morbidade decorrente de doenças respiratórias e circulatórias. Para tanto, primeiramente apresentam-se algumas características das OdF utilizando uma série de dados meteorológicos de 1984 a 2013 e, posteriormente, comparando os dados de frio com dados de internação hospitalar de uma série histórica de 15 anos.

Vale salientar que neste estudo não foi possível integrar os dados socioeconômicos nas análises, pois as tabelas de internações diárias disponibilizadas pelo Departamento de Informática do Sistema Único de Saúde (DATASUS) para São Joaquim não disponibilizam, por exemplo, a informação de renda, de escolaridade, entre outros aspectos do internado. Da mesma forma, ressalta-se que, todas as internações individuais estavam cadastradas em um único Código de Endereçamento Postal (CEP), impossibilitando a espacialização dos enfermos por setores censitários, o que também permitiria um enfoque socioeconômico se existisse diferenciação dos locais. Porém, é sabido que em SC o Planalto Sul é uma das localidades com piores índices socioeconômicos quando comparada as demais (IBGE, 2003; PNUD, 2010).

\section{CARACTERÍSTICAS DO LOCAL DE ESTUDO}

São Joaquim possui área total de $1.892 .256 \mathrm{~km}^{2}$ e está localizado no Planalto Sul catarinense, entre as paralelas $28^{\circ} 30^{\prime \prime S}$ e $28^{\circ} 00^{\prime \prime S}$ e $49^{\circ} 30^{\prime \prime} \mathrm{W}$ e 50030"W (Figura 1). A última estimativa, realizada em 2017, apontava para uma população de 26.646 pessoas (IBGE, 2017). De acordo com o Censo de $2010,29,2 \%$ da população habitava a zona rural e $70,8 \%$ a zona urbana (IBGE, 2010). Há ainda uma população flutuante no município, principalmente na época da colheita da maçã, que vai de fevereiro a maio. Isto ocorre devido à maior demanda de trabalhadores e acarreta em aumento da pressão sobre os serviços públicos (CORDEIRO, 2006). 


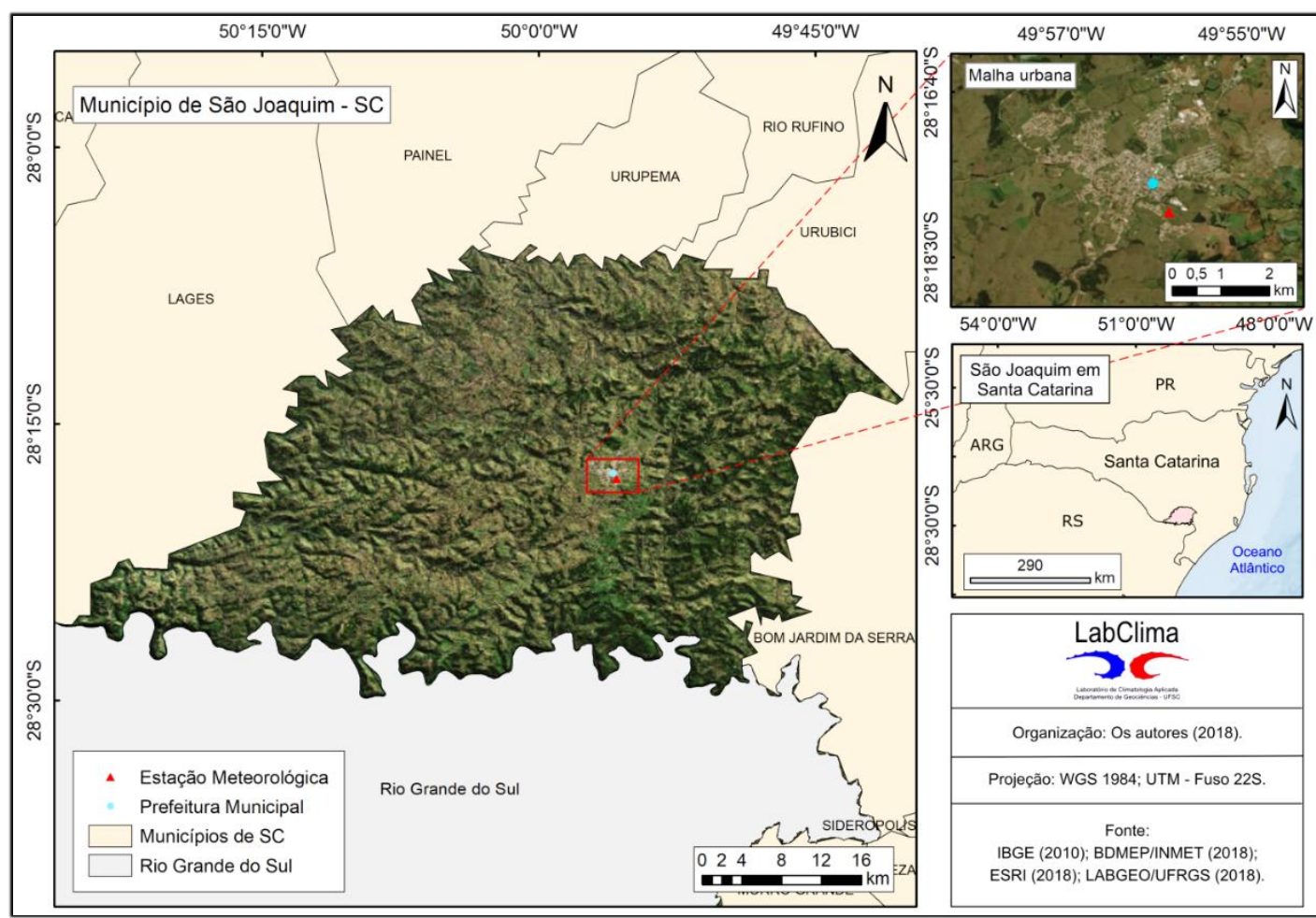

Figura 1 - Localização do município de São Joaquim e da estação meteorológica convencional.

Apenas com intuito de caracterização, segundo o IBGE (2003), em seu último mapa realizado, o índice de pobreza de São Joaquim em 2003 atingia $31,17 \%$ da população, posicionando o município dentro da classe dos mais pobres de SC. Conforme o Programa das Nações Unidas para o Desenvolvimento (PNUD) (2010), São Joaquim tem uma população vulnerável a pobreza de $29,7 \%$. Por outro lado, ainda conforme o PNUD (2010), o Índice de Desenvolvimento Humano (IDH) de São Joaquim tem evoluído nos últimos anos, passando de 0,589 em 2000 para 0,687 em 2010. Carson et al. (2006) apontam que muitas internações e mortes ocasionadas ou potencializadas pela temperatura do ar, neste caso especificamente pelo frio, podem ser evitadas. As taxas de morbidade e de mortalidade vinculadas com as temperaturas podem apontar/refletir a deficiência da saúde pública de um determinado local. Contudo, outros fatores podem contribuir para isto, dentre eles: influências ambientais; comportamentais (individuais e contextuais) e; especialmente, sociais, que em grande parte tem relação com o aspecto socioeconômico das populações.

O clima de São Joaquim é o Cfb, temperado constantemente úmido, sem estação seca e com verão fresco (KÖPPEN; GEIGER, 1936). Conforme dados do Instituto Nacional de Meteorologia (INMET) (2018), no trimestre invernal (junho a agosto) a temperatura média varia de 9,7 a $10,7^{\circ} \mathrm{C}$. Em São Joaquim ocorrem geadas, com 29 a 36 registros por ano, e precipitação de neve, com média anual de 2,7 dias (SOUZA, 1997; EPAGRI, 2004; SCHIMITZ, 2007). Tais informações evidenciam como as temperaturas são amenas em São Joaquim e 
como existem maiores condições ao frio, quando comparado ao Brasil de forma geral.

De acordo com o Cadastro Nacional de Estabelecimentos de Saúde (CNES) (2015), em São Joaquim existiam 78 profissionais ligados diretamente à saúde. O município possuía um hospital estadual que atendia parcialmente pelo SUS, mas com esfera administrativa privada. Além do hospital, no mesmo ano existiam mais seis Unidades Básicas de Saúde (UBS) (CNES, 2015).

São Joaquim tem uma grande área territorial, entretanto, sua parte urbanizada concentra-se no centro geográfico do município, abrangendo uma área relativamente pequena (DUSI, 2007); isto pode ser mais bem evidenciado na Figura 1 (acima), demonstrando como a malha urbana é restrita a uma pequena porção do território. Devido a isso, percebe-se que apesar das UBS não estarem tão distantes umas das outras, ainda assim são bem distribuídas na área urbanizada, atendendo locais distintos. Todavia, devido a grande área territorial municipal, as pessoas residentes nas localidades rurais (quase $1 / 3$ da população) podem ter dificuldade de acesso às UBS devido à distância.

No estado de SC e não diferente para São Joaquim, grande parte dos veículos de mídia geralmente atribuem ao frio somente os aspectos positivos. Exemplos disso são as reportagens e chamadas a respeito do frio intenso e da neve abordando vários quesitos, mas negligenciando o frio como um possível desastre ou, ao menos, como potencializador de problemas sociais (SILVEIRA et al., 2014). O impacto do frio extremo ou das ondas de frio é citado como desastre inclusive pelo Ministério da Integração Nacional (CASTRO et al., 2003). Em consequência disto os problemas relacionados às baixas temperaturas, principalmente entre as populações mais vulneráveis, acabam ofuscados. A tônica das reportagens é sobre a beleza da paisagem e seu apelo turístico, no midiático "Planalto das Neves" (SOUZA, 1997), que no inverno no município incrementa os ganhos do terceiro setor, como o comércio, hotéis, restaurantes e agências de turismo.

\section{MATERIAIS E MÉTODOS \\ IDENTIFICAÇÃO DAS OdF}

As OdF foram identificadas por meio da média diária da temperatura do ar compensada $(\bar{T})$, observada na estação meteorológica convencional de São Joaquim. Esta estação é administrada pela Empresa de Pesquisa Agropecuária e Extensão Rural de Santa Catarina - Centro de Informações de Recursos Ambientais e Hidrometeorologia de Santa Catarina (Epagri/Ciram). Considerouse OdF a situação em que dois ou mais dias consecutivos tiveram $\bar{T}$ inferior ao valor da temperatura climatológica diária $\left(\overline{T_{c l l}}\right)$ subtraída de dois desvios padrões $(\bar{S})$, ou seja, quando $\bar{T} \leq\left(\overline{T_{c l l}}-2 \bar{S}\right)$ (VAVRUS et al., 2006). O desvio padrão, $\bar{S}$, é dado pelo valor médio interanual dos 365 (10 de janeiro a 31 de dezembro) desvios padrões diários, que no caso de São Joaquim, é igual a $3,14^{\circ} \mathrm{C}$. A identificação das OdF foi feita para os 365 dias do ano e considerou o período de 1984 a 2013 (366 dias para anos bissextos). Em toda série apenas seis dias apresentaram falhas, mas, os mesmos não coincidiram com nenhuma onda encontrada. Os limiares diários para que um dia seja considerado frio e, possivelmente, integre uma OdF podem ser visualizados na Tabela 1. Vale 
apontar que o método foi adaptado de Vavrus et al. (2006), pois originalmente os autores aplicaram a técnica apenas para o período de inverno.

Tabela 1 - Limiares diários $\left({ }^{\circ} \mathrm{C}\right)$ separados mensalmente, São Joaquim/SC - 1984 a 2013.

\begin{tabular}{|c|c|c|c|c|c|c|c|c|c|c|c|c|}
\hline \multirow{2}{*}{ Dia } & \multicolumn{12}{|c|}{ Mês } \\
\hline & Jan & Fev & Mar & Abr & Mai & Jun & Jul & Ago & Set & Out & Nov & Dez \\
\hline 1 & 10,48 & 11,20 & 10,54 & 8,58 & 6,04 & 2,20 & 4,42 & 3,77 & 5,09 & 6,24 & 6,59 & 8,80 \\
\hline 2 & 10,13 & 10,93 & 10,59 & 8,69 & 5,70 & 3,03 & 4,27 & 3,42 & 4,28 & 6,74 & 7,18 & 9,02 \\
\hline 3 & 10,24 & 11,24 & 10,42 & 8,91 & 6,05 & 3,31 & 3,58 & 3,18 & 4,61 & 6,58 & 7,86 & 9,72 \\
\hline 4 & 10,69 & 11,27 & 10,79 & 8,43 & 5,69 & 3,44 & 3,68 & 3,40 & 4,67 & 6,71 & 7,77 & 10,01 \\
\hline 5 & 11,00 & 11,20 & 10,71 & 8,03 & 5,12 & 3,82 & 4,14 & 4,59 & 4,85 & 6,91 & 7,26 & 10,19 \\
\hline 6 & 10,91 & 11,06 & 10,54 & 8,15 & 4,25 & 3,54 & 3,18 & 5,30 & 5,64 & 6,35 & 7,55 & 10,16 \\
\hline 7 & 11,22 & 10,88 & 10,65 & 7,93 & 4,52 & 3,24 & 2,53 & 4,61 & 5,79 & 6,13 & 8,26 & 9,82 \\
\hline 8 & 11,41 & 10,74 & 11,11 & 7,87 & 4,98 & 3,62 & 2,45 & 4,58 & 5,42 & 6,09 & 8,75 & 9,65 \\
\hline 9 & 10,97 & 10,65 & 11,10 & 8,16 & 5,05 & 3,59 & 3,06 & 4,10 & 4,89 & 6,52 & 9,07 & 10,00 \\
\hline 10 & 10,44 & 10,57 & 10,45 & 8,56 & 5,02 & 2,61 & 2,81 & 2,95 & 5,51 & 6,39 & 8,36 & 10,08 \\
\hline 11 & 10,72 & 10,18 & 10,08 & 8,20 & 5,11 & 3,49 & 1,25 & 3,20 & 5,90 & 6,86 & 8,09 & 10,01 \\
\hline 12 & 10,86 & 10,38 & 10,00 & 8,37 & 4,81 & 4,08 & 1,55 & 4,64 & 5,27 & 7,20 & 7,56 & 9,95 \\
\hline 13 & 11,23 & 10,28 & 10,15 & 7,91 & 4,54 & 4,41 & 1,93 & 5,10 & 5,33 & 7,72 & 7,88 & 9,57 \\
\hline 14 & 11,23 & 10,20 & 9,76 & 7,91 & 4,08 & 4,47 & 3,14 & 4,87 & 5,16 & 7,76 & 8,51 & 9,89 \\
\hline 15 & 10,95 & 10,51 & 9,73 & 8,14 & 4,38 & 4,37 & 2,96 & 4,73 & 4,05 & 7,85 & 8,84 & 10,04 \\
\hline 16 & 10,46 & 10,63 & 9,59 & 7,98 & 4,43 & 3,91 & 3,81 & 3,66 & 3,88 & 7,44 & 8,96 & 10,24 \\
\hline 17 & 10,52 & 10,69 & 9,73 & 7,63 & 4,33 & 3,79 & 3,97 & 3,97 & 4,51 & 6,93 & 8,11 & 9,37 \\
\hline 18 & 10,54 & 10,57 & 9,72 & 7,12 & 4,25 & 3,84 & 3,38 & 4,11 & 4,80 & 5,92 & 7,76 & 9,57 \\
\hline 19 & 10,43 & 10,89 & 9,53 & 6,74 & 4,45 & 3,64 & 2,83 & 5,36 & 5,09 & 6,82 & 8,55 & 10,32 \\
\hline 20 & 10,49 & 11,40 & 9,51 & 6,66 & 4,71 & 3,64 & 3,38 & 4,76 & 5,21 & 7,19 & 8,50 & 10,31 \\
\hline 21 & 10,55 & 11,32 & & 6,73 & 4,14 & 2,88 & 2,65 & 4,06 & 5,33 & 6,92 & 8,92 & 10,45 \\
\hline 22 & 10,50 & 11,03 & 9,48 & 7,12 & 4,14 & 3,02 & 1,43 & 4,73 & 5,75 & 7,03 & 8,97 & 10,62 \\
\hline 23 & 10,87 & 10,90 & 9,69 & 7,51 & 3,87 & 3,88 & 0,65 & 5,23 & 5,41 & 7,42 & 9,55 & 10,17 \\
\hline 24 & 11,29 & 10,58 & 9,60 & 7,49 & 4,14 & 3,63 & 1,56 & 5,44 & 4,14 & 7,25 & 9,56 & 10,50 \\
\hline 25 & 10,95 & 10,64 & & 7,34 & 3,63 & 3,18 & 2,40 & 5,56 & 3,39 & 7,61 & 9,34 & \\
\hline 26 & 10,48 & 11,30 & 8,73 & 6,98 & 3,72 & 3,31 & 3,28 & 5,54 & 3,93 & 7,47 & 9,59 & 10,59 \\
\hline 27 & 10,69 & 11,03 & 8,96 & 6,32 & 3,75 & 3,06 & 3,27 & 4,85 & 4,10 & 7,22 & 8,99 & 10,48 \\
\hline 28 & 11,37 & 11,00 & 8,98 & 6,66 & 4,12 & 3,42 & 3,39 & 4,65 & 4,09 & 7,90 & 8,50 & 10,12 \\
\hline 29 & 11,51 & 11,07 & 9,09 & 6,47 & 3,55 & 3,59 & 3,68 & 5,73 & 4,69 & 8,49 & 9,11 & 10,41 \\
\hline 30 & 11,64 & & 9,25 & 5,75 & 2,62 & 4,11 & 3,24 & 6,08 & 5,48 & 8,97 & 9,03 & 10,47 \\
\hline 31 & 11,62 & & 9,45 & & 2,12 & & 3,40 & 6,03 & & 7,87 & & 10,70 \\
\hline
\end{tabular}

\section{DOENÇAS ANALISADAS}

Foram analisadas as doenças que normalmente estão relacionadas com a ocorrência de frio, ou seja, as doenças relacionadas aos aparelhos respiratório e circulatório. Os dados foram obtidos do DATASUS - Ministério da Saúde. A disponibilidade destes dados começa em 1998, mas a análise de comparação com as OdF é realizada para o período de 1999 a 2013 (15 anos), pois em 1998 não houve registro de OdF.

As doenças do aparelho respiratório e aparelho circulatório foram subdivididas em seis, conforme apresentado na Lista de Tabulação para Morbidade do SUS, pertencentes à Classificação Internacional de Doenças (CID 10) (Tabela 2). 
Tabela 2 - Lista de doenças selecionadas para análise da relação com as OdF.

\begin{tabular}{ll}
\hline \multicolumn{2}{c}{ Informações de saúde (TABNET) } \\
\hline \multicolumn{1}{c}{ Código CID-10 } & \multicolumn{1}{c}{ Dpidemiológicas e morbidade } \\
J45/J46 & Asma respiratórias \\
J09/J11 & Influenza (gripe) \\
J12-J18 & Pneumonia \\
Código CID-10 & \multicolumn{1}{c}{ Doenças circulatórias } \\
I21/I22 & Infarto do miocárdio \\
I64 & Acidente Vascular Cerebral (AVC) \\
I20, I23-I25 & Outras Doenças Isquêmicas do Coração (DIC) \\
\hline
\end{tabular}

Fonte: SIH/SUS, 2015.

As DIC englobam problemas de saúde conhecidos como: angina pectoris, algumas complicações atuais subsequentes ao infarto agudo do miocárdio, outras doenças isquêmicas agudas do coração e doença isquêmica crônica do coração (OMS, 2008; SIH/SUS, 2015). Contabilizaram-se internações diárias por local de residência e por local de internação. A contabilização do número de internações foi realizada durante a ocorrência da OdF e por mais dez dias após o último dia do evento, conforme proposto por Silva (2014). Em alguns casos os dez dias posteriores ao término da OdF foram sobrepostos a outra OdF.

\section{ANÁLISE ESTATÍSTICA}

A avaliação da associação entre internações hospitalares e o frio foi realizada através dos cálculos de coeficiente de correlação ( $r$ ) que, segundo Morettin e Bussab (2013), mede a intensidade e a direção da relação linear entre duas variáveis quantitativas. A decisão entre correlação estatística significativa foi tomada pela aplicação do deste t de Student; ou seja, quando o $p$-valor do teste for menor ou igual ao nível de significância se aceita a hipótese de correlação significativa, portanto, a significância de $r$ depende do tamanho da amostra (ROGERSON, 2012). A significância estatística da dependência entre frio e internações hospitalares foi avaliada, a partir da regressão linear simples, pelo coeficiente de determinação $\left(r^{2}\right)$ e análise da variância, ao nível de significância $a=5 \%$. Segundo Barbetta et al. (2010), pode-se verificar o percentual da variância total que é explicada pela reta obtida de um modelo de regressão linear, utilizando-se o $r^{2}$, ou seja, se a porção da variância total explicada pela regressão é estatisticamente significativa. Conforme Rogerson (2012, p. 201), a regressão linear examina o relacionamento entre uma variável dependente e uma variável explicativa independente. Esta análise parte do princípio de que há um relacionamento linear entre a variável dependente/resposta $(y)$ e a variável independente/explicativa $(x)$. A regressão linear é a espécie de regressão caracterizada pelo fato de ser linear a forma escolhida para a equação de regressão; ela pode ser simples (uma variável independente) ou múltipla (duas ou mais variáveis independentes) (RODRIGUES, 1970, p. 257). O coeficiente de determinação ( $\left.r^{2}\right)$ explica quanto a variável independente, no caso específico: frio, explica o comportamento da variável dependente (internações hospitalares). $0 r^{2}$ é uma medida de ajustamento de um modelo estatístico linear, como a própria regressão linear, esta medida varia de 0 a 1 , explanando, em porcentagem, quanto o modelo consegue elucidar os valores observados; quanto maior, mais explicativo é o modelo, melhor se ajustando a amostra (ROGERSON, 2012). A decisão da 
significância estatística é tomada pelo tamanho do $p$-valor, ou seja, quando este for menor ou igual ao nível de significância adotado no teste.

Para cada ano, foi contabilizado o número de OdF ( $n \mathrm{OdF}$ ) e o somatório de dias com OdF ( $\left.\sum d O d F\right)$, ou seja, a soma da duração de todas as OdF identificadas no ano. Além disso, para estimar a intensidade das OdF no ano, foi calculada a média da $\bar{T}$ de todos os dias com OdF no ano, convencionada aqui por $T_{m}$. A morbidade hospitalar foi caracterizada pelo número de internações por ano $\left(\sum I\right)$ devido à pneumonia, asma, gripe, AVC, DIC ou infarto do miocárdio. As comparações foram realizadas ano a ano, no período de 1999 a 2013, por meio do cálculo de $r$ e $r^{2}$ entre $\Sigma d O d F$ e $\Sigma I$ e entre $T_{m}$ e $\Sigma I$, separadamente por doença

\section{RESULTADOS E DISCUSSÕES}

\section{CARACTERIZAÇÃO DAS OdF EM SÃO JOAQUIM}

Foram identificadas 105 OdF ao longo dos 30 anos analisados, com média de 3,5 por ano. Eventos com duração de dois dias foram os que mais ocorreram, com 66 casos (62,8 \%) no total (Tabela 3 ). As OdF com duração de três dias consecutivos ocorreram 27 vezes $(25,7 \%)$, enquanto aquelas que duraram quatro dias foram apenas sete $(6,6 \%)$. Notamos ainda na Tabela 3 que, quatro OdF duraram cinco dias $(3,8 \%)$ e uma única teve a duração de seis dias $(1,1 \%)$, sendo esta a mais longa de toda série, ocorrida entre os dias 25 e 30 de agosto de 2003.

Tabela 3 - Ocorrência das OdF por data, São Joaquim/SC, 1984 a 2013.

\begin{tabular}{|c|c|c|}
\hline Ano & Período de ocorrência da OdF & No OdF \\
\hline 1984 & $24-25 / 04 ; 27-28 / 06 ; 14-17 / 08 ; 25-28 / 08 ; 18-19 / 12$ & 5 \\
\hline 1985 & $30 / 04$ a $02 / 05 ; 06-09 / 06 ; 10-11 / 07 ; 01-02 / 09$ & 4 \\
\hline 1986 & $23-24 / 10$ & 1 \\
\hline 1987 & $10-12 / 03 ; 21-22 / 05 ; 15-17 / 06 ; 23-24 / 06 ; 17-21 / 08 ; 30-31 / 08$ & 6 \\
\hline 1988 & $08-09 / 04 ; 04-05 / 06 ; 14-15 / 06 ; 11-12 / 07 ; 24-25 / 07$ & 5 \\
\hline 1989 & $05-07 / 07$ & 1 \\
\hline 1990 & $21-22 / 05 ; 10-11 / 06 ; 15-16 / 06 ; 20-22 / 07 ; 27-31 / 07 ; 23-24 / 08 ; 28-29 / 08 ; 13-14 / 09$ & 8 \\
\hline 1991 & $01-03 / 08$ & 1 \\
\hline 1992 & $22-23 / 07 ; 01-02 / 08 ; 08-09 / 08 ; 04-05 / 11$ & 4 \\
\hline 1993 & $27-28 / 05 ; 09-10 / 06 ; 18-19 / 06 ; 13-15 / 07 ; 30 / 07$ a $01 / 08$ & 5 \\
\hline 1994 & $25-27 / 06 ; 08-10 / 07 ; 08-09 / 09$ & 3 \\
\hline 1995 & $21-23 / 04 ; 18-19 / 05 ; 04-05 / 08 ; 19-20 / 09 ; 28-30 / 10$ & 5 \\
\hline 1996 & $17-18 / 04 ; 28-30 / 06 ; 09-11 / 09$ & 3 \\
\hline 1997 & $07-08 / 06$ & 1 \\
\hline 1998 & & 0 \\
\hline 1999 & $16-18 / 04 ; 19-21 / 05 ; 05-06 / 06 ; 14-16 / 08 ; 02-04 / 10 ; 09-10 / 11$ & 6 \\
\hline 2000 & $11-14 / 06 ; 16-20 / 07 ; 23-24 / 07 ; 11-12 / 08$ & 4 \\
\hline 2001 & $20-22 / 06 ; 27-28 / 07$ & 2 \\
\hline 2002 & $31 / 08$ a $02 / 09 ; 07-08 / 09$ & 2 \\
\hline 2003 & $11-12 / 04 ; 09-10 / 08 ; 25-30 / 08 ; 09-10 / 09$ & 4 \\
\hline 2004 & $27-28 / 05 ; 01-13 / 06 ; 07-08 / 08 ; 11-12 / 09$ & 4 \\
\hline 2005 & $18-19 / 07 ; 24-25 / 08 ; 12-13 / 09$ & 3 \\
\hline 2006 & $29-31 / 07 ; 19-21 / 08 ; 28-29 / 08 ; 03-06 / 09$ & 4 \\
\hline 2007 & $08-09 / 05 ; 29-30 / 05 ; 28-29 / 07 ; 27-28 / 08 ; 06-07 / 09$ & 5 \\
\hline 2008 & $15-16 / 06 ; 21-23 / 06 ; 29-30 / 08 ; 06-07 / 09 ; 03-04 / 12$ & 5 \\
\hline 2009 & $02-03 / 06$ & 1 \\
\hline 2010 & $13-16 / 07 ; 02-06 / 08 ; 14-15 / 08$ & 3 \\
\hline 2011 & $26-27 / 06 ; 03-06 / 07 ; 02-04 / 08 ; 20-22 / 08 ; 31 / 08$ a $01 / 09$ & 5 \\
\hline 2012 & $30 / 04$ a $01 / 05 ; 07-08 / 06$ & 2 \\
\hline 2013 & $22-24 / 07 ; 13-15 / 08 ; 26-28 / 08$ & 3 \\
\hline Total & & 105 \\
\hline
\end{tabular}


Durante o período de ocorrência das OdF, as menores temperaturas médias diárias do ar $(\bar{T})$ ocorreram nos dias 02 de agosto de 1991, registrando $-4,8^{\circ} \mathrm{C}$, e 13 de julho de 2000 , com $-4,7^{\circ} \mathrm{C}$. Em ambos os casos, a $\bar{T}$ ficou bem abaixo do seu limiar, conforme método de Vavrus et al. (2006). Em 1998 não existiu ocorrência de OdF e em 1990 incidiu o maior número de registros, com oito eventos, seguido por 1987 e 1999, ambos com seis eventos cada (Figura 2). Nota-se que a tendência definida por meio de modelo linear simples aponta para uma ligeira diminuição do número de OdF ao longo dos 30 anos (Figura 2).

No ano de 1998 não foi registrada a ocorrência de OdF em São Joaquim, assim como em Curitiba no mesmo ano (SILVEIRA et al., 2017). Em ambos os casos a falta de ocorrência não foi acarretada pela indisponibilidade ou falta de dados, apenas pelas condições atmosféricas. É possível que isto tenha relação com o El Niño, a fase positiva do El Niño-Oscilação Sul (ENOS), que em 1997 foi classificado como muito forte e, sabe-se que o fenômeno apresenta sua influência com maior evidência no ano seguinte, neste caso potencializando anomalias positivas de temperatura em 1998, o que pode ter afetado na não ocorrência de OdF (Figura 2) (GGWS, 2018). Firpo et al. (2012, p. 103) afirmam que, para o Rio Grande do Sul, as OdF em anos influenciados pela La Niña são mais recorrentes, já em anos de El Niño a tendência é a diminuição dos registros. Mesmo sabendo que o efeito do ENOS não é tão intenso na temperatura do ar quanto na precipitação, existe sim influência, sendo mais forte durante o inverno no Sul do Brasil e com anomalias positivas de temperatura durante o El Niño (BARROS et al., 2002; GRIMM, 2009, p. 368).

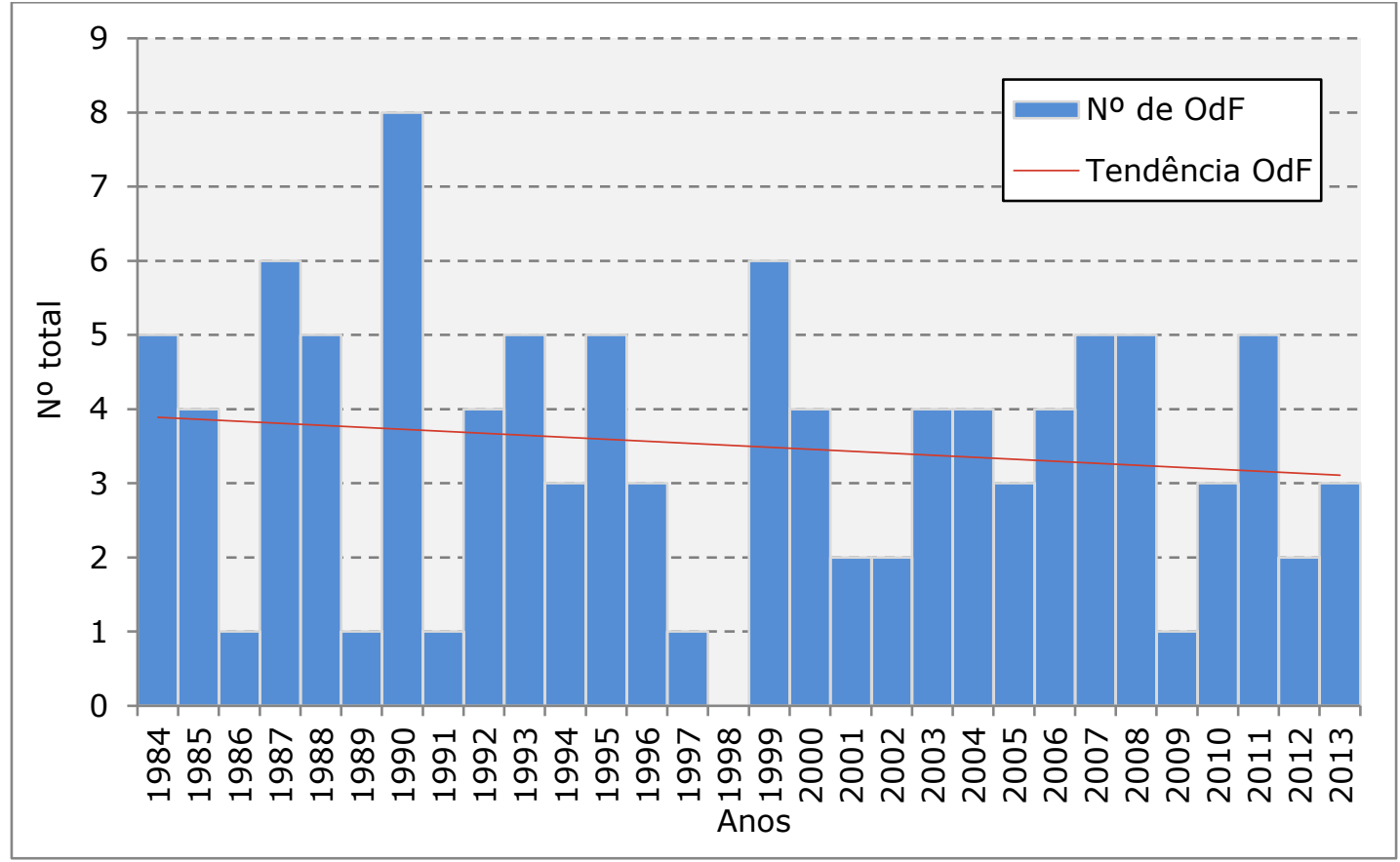

Figura 2 - Ocorrência das OdF por ano em São Joaquim e tendência definida por meio de modelo linear simples. 
A avaliação mensal das OdF mostra claramente que a maior frequência ocorre durante o inverno. O mês com mais OdF foi agosto, com 28 eventos (26,6\%), seguido de junho e julho, com 21 (20\%) e 20 eventos (19\%), respectivamente. Nas estações de transição também ocorrem OdF, com 12 eventos $(11,4 \%)$ em setembro e oito eventos em cada um dos meses de abril e maio $(7,6 \%)$ (Figura 3 ). Uma das características do método de identificação proposto por Vavrus et al. (2006), que assume um desvio padrão constante obtido da média dos 365 dias do ano, é o fato da maior frequência de OdF aparecer nos meses climatologicamente mais frios. Embora isso pareça óbvio, quando se utiliza desvio padrão diário e não uma média anual, são identificadas "ondas de frio" em meses mais quentes, os quais, criteriosamente, deveriam ser chamados de períodos "relativamente mais frios" para a época do ano.

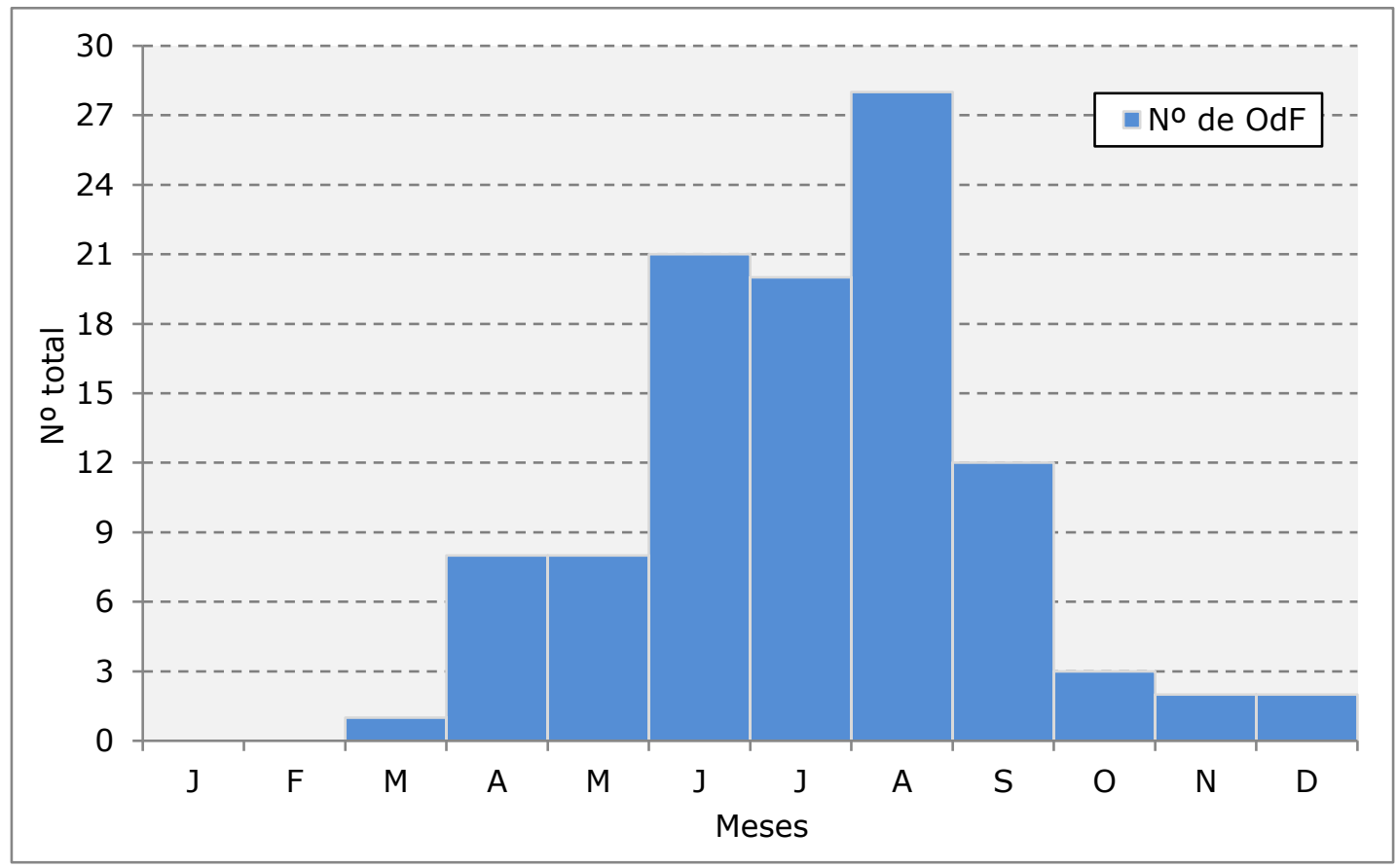

Figura 3 - Número mensal de OdF em São Joaquim, durante o período de 1984 a 2013.

Entre as 105 OdF registradas, 65,7\% ocorreram no período invernal, seguido pelo outono e primavera, ambas com $16,2 \%$ de ocorrência. No período veranil foi registrado apenas $1,9 \%$. Portanto, as OdF em São Joaquim são mais frequentes no inverno, todavia, também ocorrem com relativa frequência nos dois meses que antecedem e no mês seguinte ao inverno. Os registros de OdF em abril e maio são decorrentes das primeiras incursões de frentes frias e massas de ar polar no ano. No estado de SC a frequência de frentes frias oscila entre 25 e 35 por ano, sendo mais frequentes de maio a outubro (MONTEIRO, FURTADO, 1995; CAVALCANTI, KOUSKY, 2009). 


\section{IMPACTO DAS OdF NAS INTERNAÇÕES HOSPITALARES}

Dentro do período de abrangência e de efeito das OdF, a morbidade por doenças do aparelho respiratório é significativamente maior em relação à morbidade por doenças do aparelho circulatório (Tabela 4). As internações por doenças no aparelho respiratório, quando agrupadas, somam 604 casos (85,9\%) no período de 1999 a 2013. Já, para o mesmo período, as internações por doenças do aparelho circulatório agrupadas somam 99 casos $(14,1 \%)$, ou seja, 505 casos a menos que as doenças respiratórias.

Destacam-se as internações por pneumonia, que contabilizaram mais registros do que todas as outras somadas (64,1\%). Em 2011 foram registrados 66 casos de internações durante as cinco OdF em São Joaquim (Tabela 4). A influenza (gripe) foi a segunda maior causa de internação hospitalar, com 81 internações $(11,5 \%)$. Já a asma foi a terceira, com 72 internações $(10,2 \%)$. Todavia, estas internações foram mais distribuídas ao longo do tempo.

Entre as internações por doenças circulatórias, os casos de AVC foram os mais recorrentes, somando 43 registros (6,1\%). Nos anos de 1999, 2001, 2005, 2006 e 2007 não foram registradas internações. Os casos de DIC aparecem como a segunda maior causa de morbidade entre as doenças circulatórias, com 36 casos $(5,1 \%)$. As internações por infarto do miocárdio, dentre as seis doenças analisadas, foram aquelas com menor registro, aproximadamente $3 \%$ do total, sendo 2003 o ano com mais internações (Tabela 4).

Tabela 4 - Dados das ondas de frio e internações por ano, São Joaquim/SC, 1999 a 2013.

\begin{tabular}{|c|c|c|c|c|c|c|c|c|c|}
\hline \multirow{2}{*}{ Ano } & \multirow{2}{*}{$n O d F$} & \multirow{2}{*}{$\sum d \mathrm{OdF}$} & \multirow{2}{*}{$T_{m}\left({ }^{\circ} \mathrm{C}\right)$} & \multicolumn{6}{|c|}{$\sum I$} \\
\hline & & & & Pneumonia & Asma & Gripe & AVC & DIC & Infarto do miocárdio \\
\hline 1999 & 6 & 16 & 3,4 & 58 & 9 & 0 & 0 & 5 & 1 \\
\hline 2000 & 4 & 13 & $-0,3$ & 39 & 18 & 0 & 6 & 1 & 0 \\
\hline 2001 & 2 & 5 & 1,4 & 9 & 10 & 0 & 0 & 2 & 1 \\
\hline 2002 & 2 & 5 & 3,0 & 12 & 6 & 0 & 1 & 2 & 0 \\
\hline 2003 & 4 & 12 & 3,5 & 18 & 7 & 1 & 6 & 5 & 5 \\
\hline 2004 & 4 & 8 & 3,1 & 34 & 7 & 0 & 1 & 4 & 2 \\
\hline 2005 & 3 & 6 & 2,8 & 11 & 5 & 0 & 0 & 1 & 0 \\
\hline 2006 & 4 & 12 & 2,5 & 22 & 1 & 0 & 0 & 3 & 1 \\
\hline 2007 & 5 & 8 & 2,3 & 22 & 7 & 19 & 0 & 5 & 1 \\
\hline 2008 & 5 & 11 & 4,3 & 10 & 1 & 26 & 5 & 2 & 1 \\
\hline 2009 & 1 & 2 & 2,1 & 2 & 0 & 9 & 2 & 0 & 0 \\
\hline 2010 & 3 & 11 & 1,9 & 58 & 0 & 0 & 4 & 1 & 1 \\
\hline 2011 & 5 & 14 & 1,9 & 66 & 0 & 0 & 8 & 0 & 3 \\
\hline 2012 & 2 & 4 & 3,2 & 35 & 1 & 26 & 6 & 3 & 0 \\
\hline 2013 & 3 & 9 & 1,8 & 55 & 0 & 0 & 4 & 2 & 4 \\
\hline Total & 53 & 136 & & 451 & 72 & 81 & 43 & 36 & 20 \\
\hline
\end{tabular}

Fonte: SIH/SUS, 2015.

É interessante informar que, das 703 internações totalizadas sob influência das OdF, $88 \%$ foram de joaquinenses que se internaram em seu próprio município; $7 \%$ de joaquinenses que se internaram em outros municípios e; $5 \%$ de indivíduos não joaquinenses que se internaram em São Joaquim. Todos de São Joaquim que se internaram fora das unidades locais procuraram 
outras dentro de SC; já dos não joaquinenses que se internaram em São Joaquim, apenas dois não residiam no estado, um sendo proveniente do Rio Grande do Sul e outro de São Paulo. Acrescenta-se que os dados de internações disponibilizados e contabilizados pelo DATASUS não englobam atendimentos em unidades privadas/particulares, exceto se a mesma prestar atendimento ao SUS (MS, 2004, p. 11). Acredita-se que o baixo percentual de internações provenientes de pessoas que residiam fora de São Joaquim tenha ocorrido devido ao fato de que, em geral, os turistas ficam pouco tempo sob influência das condições de frio intenso ou de forma não contínua; geralmente estão com vestimentas ideais e em edificações mais bem preparadas para o frio e; se por ventura ficarem enfermos, possuem condições de procurar unidades de saúde particulares, devido as condições financeiras ou por possuirem planos de saúde. Já com relação aos trabalhadores sazonais que vão ao município principalmente na época da colheita da maçã, presume-se que os mesmos não tenham impactado tanto no percentual de forasteiros que se internam em São Joaquim, pois a temporada da colheita vai de fevereiro a maio, período não tão rigoroso quanto ao frio; ou porque a maioria é proveniente de SC ou dos estados do Sul, o que de certa forma faz com que o trabalhador seja aclimatado às condições de temperaturas mais amenas e também mais adaptado, diminuindo a possibilidade de adoecimento (EDLER, 2010); diferentemente se fossem de estados do Centro-Oeste ou Nordeste, por exemplo.

Notamos na Tabela 5 que há correlação estatisticamente significativa $(r=0,63)$ entre o número de dias com OdF e o número de internações por pneumonia ( $\sum d \mathrm{OdF}$ versus $\Sigma I$ ). As demais doenças apresentaram valores de correlação inferiores a 0,514, mostrando associação estatisticamente não significativa entre a ocorrência de frio e a morbidade decorrente de asma, gripe, AVC, DIC e infarto do miocardio. O valor 0,514 é o valor mínimo, para que uma amostra $\mathrm{n}=15$, tenha o $r$ singnificativo ao nível de $5 \%$. Pode-se chegar à mesma conclusão analisando-se o tamanho do $p$-valor na Tabela 5. A única variável que apresentou um $p$-valor menor que o nível de significância adotado $(a=0,05$ ou $5 \%$ ) foi a pneumonia.

No caso da relação entre a intensidade média das OdF, representada pela média anual da $\bar{T}$ nos dias com OdF $\left(T_{m}\right)$, e a soma de internações $(\Sigma I)$ devido a qualquer uma das doenças ( $T_{m}$ versus $\sum I$, apresentada na Tabela 5 ), nenhuma das análises se mostrou estatisticamente significativa. Ainda assim, a correlação $(r)$ que foi menor para pneumonia, aparece maior $(r \approx 0,4)$ para asma, gripe e DIC em relação à avaliação anterior.

A qualidade do ajuste do modelo de regressão linear simples foi avaliado pela análise da variância (ANOVA) ao nível de significância de $a=5 \%$. Percebese, na Tabela 5, que o $p$-valor, para todas as regressões testadas neste estudo, foi superior a 0,05, sugerindo não haver dependência linear, exceto entre 0 somatório anual de dias com OdF e as internações por pneumonia, em que o $p$ valor foi 0,01193 . Este resultado além de indicar que a pneumonia é o mais evidente impacto causado pelo frio, também sugere que a ocorrência do frio é mais impactante em invernos com muitos dias de frio, mesmo que não sejam registradas temperaturas baixas extremas. Ou seja, os invernos que apresentam muitos dias e noites frias, decorrentes da incursão de muitas massas de ar frio, são mais impactantes à saúde pública do que os invernos com apenas uma ou duas massas de ar frio, ainda que estas tenham sido com temperaturas extremamente baixas. 
Tabela 5 - Relações entre a soma anual de dias com OdF ( $\Sigma d O d F)$ e o número anual de internações $\left(\sum I\right)$ e entre a média anual da $\bar{T}$ nos dias com OdF $\left(T_{m}\right)$ e o número anual de internações $\left(\sum I\right)$, separadamente por doenças.

\begin{tabular}{lccccc}
\hline & \multicolumn{5}{c}{$\sum d$ OdF versus $\sum I$} \\
\hline \multicolumn{1}{c}{ Doença } & $\mathrm{r}$ & $\mathrm{r}^{2}$ & $p$-valor & Coef. linear (a) & Coef. angular (b) \\
\hline Pneumonia & 0,62939 & 0,39613 & 0,01193 & 0,642816256 & 3,245277619 \\
Asma & 0,19814 & 0,03926 & 0,47902 & 2,534630796 & 0,249856898 \\
Gripe & 0,28879 & 0,0834 & 0,29653 & 11,72123641 & $-0,697195192$ \\
AVC & 0,29836 & 0,08902 & 0,28008 & 0,993131082 & 0,206639954 \\
DIC & 0,23975 & 0,05748 & 0,38944 & 1,48139668 & 0,101316543 \\
Inf. do miocárdio & 0,40477 & 0,16384 & 0,1345 & $-0,054951345$ & 0,153119634 \\
\hline \multicolumn{1}{c}{$\quad T_{m}$ versus $\sum I$} \\
\hline \multicolumn{1}{c}{ Doença } & $\mathrm{r}$ & $\mathrm{r}^{2}$ & $p$-valor & Coef. linear (a) & Coef. angular (b) \\
\hline Pneumonia & 0,23616 & 0,05577 & 0,39678 & 41,37115299 & $-4,592001124$ \\
Asma & 0,42757 & 0,18282 & 0,11189 & 9,80531687 & $-2,033212304$ \\
Gripe & 0,41369 & 0,17114 & 0,12531 & $-3,87146355$ & 3,766165911 \\
AVC & 0,12992 & 0,01688 & 0,64445 & 3,70198826 & $-0,339316409$ \\
DIC & 0,46765 & 0,2187 & 0,07878 & 0,56532498 & 0,745264269 \\
Inf. do miocárdio & 0,13093 & 0,01714 & 0,64185 & 0,87353324 & 0,18677563 \\
\hline
\end{tabular}

Portanto, apenas a morbidade por pneumonia se correlacionou significativamente com as OdF. O modelo de regressão ajustado (apresentado no canto superior esquerdo da Figura 4), ao nível de $a=5 \%$ de significância, explicou que $39 \%$ das internações podem ser atribuídas ao frio ( $r^{2}-3^{a}$ coluna Tabela 5). Nenhuma das outras enfermidades tiveram significância ao nível de $a=5 \%$, rigor adotado. Dessa forma, os outros $61 \%$ devem-se a outras causas não analisadas neste estudo, como, por exemplo: renda per capita, material da habitação, escolaridade, número de pessoas por residência, acesso aos serviços de saúde, posse de aquecedores, falta de vestimentas adequadas, atividade ocupacional, entre outras.

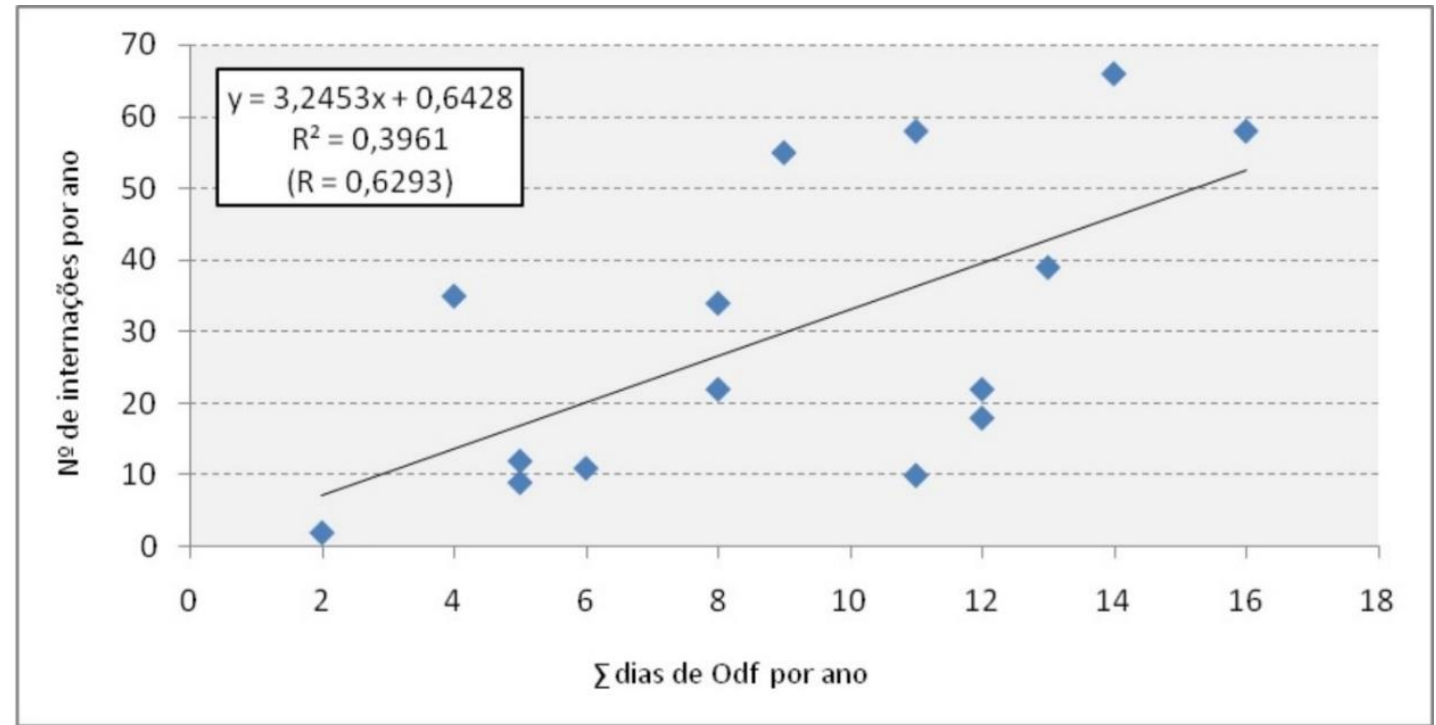

Figura 4 - Dispersão dos pontos ( $n^{\circ}$ de internações por pneumonia e soma dos dias de OdF), São Joaquim/SC, 1999 a 2013. 
Além desses, muitos outros aspectos influenciam o resultado de morbidade por pneumonia. Contudo, supomos que a pneumonia seja o impacto mais claro da ocorrência de frio pelo fato de ser uma doença grave, para a qual dificilmente uma pessoa com esta enfermidade deixe de procurar atendimento nas unidades básicas de saúde ou hospitais.

\section{CONCLUSÕES}

Constatou-se neste estudo que São Joaquim apresenta significativa ocorrência de OdF e temperaturas do ar realmente baixas para os padrões nacional e estadual. Os episódios de OdF se distribuem em quase todos os meses, com maior frequência e duração no inverno. Apesar de apresentar um decréscimo nas OdF nos últimos anos, tomando como base a década de 1980, ainda assim o município registra uma quantidade considerável de eventos $(3,5$ OdF/ano). A média de OdF em São Joaquim é superior à média de Curitiba, por exemplo, que registra três episódios por ano (SILVEIRA, 2017, p. 2166); esta que é considerada a capital mais fria do país (MENDONÇA; DANNI-OLIVEIRA, 2007). Isto demonstra a importância de monitorar estes casos e estar atento aos impactos que as OdF podem ocasionar na saúde pública.

O impacto do frio na saúde pública de São Joaquim foi mais claramente representado pelas internações decorrentes de pneumonia. Para esta doença, as OdF explicam 39\% dos casos de internações. Os resultados também indicam que invernos com muitos dias/noites de frio podem ser mais impactantes que invernos com poucas incursões de massas de ar frio, mesmo que estas sejam extremas e causem temperaturas bastante baixas.

Apesar das dificuldades que estudos desta natureza apresentam para quantificar os impactos do frio à saúde pública, sejam por motivos inerentes aos limites dos dados ou a aplicação espacial da pesquisa, acredita-se que resultados como os encontrados aqui são importantes para a criação ou aprimoramento de políticas públicas, assim como, no âmbito mais pontual, na otimização de recursos humanos e materiais em hospitais e unidades de saúde.

\section{AGRADECIMENTOS}

Os autores agradecem a Epagri/Ciram pelo fornecimento dos dados meteorológicos utilizados. Ao Programa de Pós-Graduação em Geografia (PPGG) da UFSC, berço desta pesquisa por meio de uma dissertação já concluída. Além destes, ao CNPq, pela bolsa de mestrado do 10 autor ( ${ }^{\circ}$ 130710/2014-8) e a CAPES pela atual bolsa (no 1696632) do mesmo, ao LabClima e ao GEDN, ambos do Departamento de Geociências da UFSC. Os autores agradecem grandemente a contribuição da Prof. ${ }^{a}$ Dr. ${ }^{a}$ Magaly Mendonça (in memoriam) por muito ter contribuído na construção deste artigo e em todas as discussões que antecederam esta e outras pesquisas. O $1^{0}$ autor, monitor da disciplina de climatologia quando ministrada pela Prof.a Magaly na UFSC, seu orientado de Trabalho de Conclusão de Curso, de mestrado e de entrada no doutorado; agradece todo seu compartilhamento de experiências acadêmicas, aprendizados e vivências. Infelizmente não foi possível fazer com que a professora visse esta pesquisa publicada, seu adoecimento e falecimento, fizeram com que o luto adiasse sua submissão. 


\section{REFERÊNCIAS BIBLIOGRÁFICAS}

BARBETTA, P.A.; REIS, M.M.; BORNIA, A.C. Estatística para cursos de engenharia e informática. 3. ed. São Paulo: Atlas, 2010. 338p.

BARNETT, A.G.; HAJAT, S.; GASPARRINI, A.; ROCKLÖV, J. Cold and heat waves in the United States. Environmental Research, v. 112, p. 218-224, 2012.

BARROS, V.R.; GRIMM, A.M.; DOYLE, M.E. Relationship between temperature and circulation in Southeastern South America and its Influence from El Niño and La Niña events. Journal of the Meteorological Society of Japan, v. 80, n. 1, p. 21-32, 2002.

BITENCOURT, D.P.; FUENTES, M.V.; LEITE, R.A.O.; POLETTO, M.D.L. Associação de variáveis meteorológicas com os afastamentos do trabalho devido a doenças respiratórias: um estudo entre trabalhadores dos correios de Santa Catarina. Revista Brasileira de Saúde Ocupacional, v. 34, n. 120, p. 139-149, 2009.

BRASIL. Ministério da Saúde. Exposição ao frio pode agravar doenças respiratórias. Portal Brasil/Portal da Saúde, 2014. Disponível em: <http://www.brasil.gov.br/saude/2014/04/exposicao-ao-frio>. Acesso em: 03 fev. 2018.

CARSON, C.; HAJAT, S.; ARMSTRONG, B.; WILKINSON, P. Declining vulnerability to temperature-related mortality in London over the 20th century. American Journal of Epidemiology, v. 164, n. 1, p. 77-84, 2006.

CASTRO, A.L.C.; CALHEIROS, L.B.; CUNHA, M.I.R.; BRINGEL, M.L.N.C. Manual de Desastres - Desastres Naturais. Ministério da Integração Nacional - MI, Brasília, v. 1, 2003.

CAVALCANTI, I.F.A.; KUOSKY, V.E. Frentes frias sobre o Brasil. In: Tempo e Clima no Brasil. Iracema F.A. Cavalcanti [et al.] organizadores. São Paulo: Oficina de Textos, 2009, Cap. 9, p. 135-147.

CNES - Cadastro Nacional de Estabelecimentos de Saúde. CNESNet - Secretaria de Atenção à Saúde - DATASUS. FCES - Ficha do Estabelecimento de Saúde. 2015.

Disponível

em: <http://cnes.datasus.gov.br/Lista_Es_Nome.asp?VTipo=0>. Acesso em: 22 mai. 2018.

CORDEIRO, W.C. A vitinicultura em São Joaquim - SC: uma nova atividade no município. Dissertação (Mestrado em Agroecossistemas) - Centro de Ciências Agrárias, Universidade Federal de Santa Catarina Florianópolis, Florianópolis, 2006.

DUSI, L. Conflitos de uso do solo na gestão ambiental de bacias hidrográficas: BH Urubici. Florianópolis. Dissertação (Mestrado em Engenharia Ambiental) Centro Tecnológico, Universidade Federal de Santa Catarina. Florianópolis, 163 p., 2007.

EDLER, F. Medicina tropical: uma ciência entre a Nação e o Império. Diálogos, DHI/PPH/UEM, v. 14, n. 2, p. 305-325, 2010.

EPAGRI - Empresa de Pesquisa Agropecuária e Extensão Rural de Santa Catarina. Organização dos 1.500 produtores é prioridade. In: S.C. Agricultura e Pesca. Edição especial Gerência Regional de São Joaquim. Florianópolis: ano 2, no 8, 2004. 
FANTE, K.P.; ARMOND, N.B. Ondas de frio e enfermidades respiratórias: análise na perspectiva da vulnerabilidade climática. Revista do Departamento de Geografia, n. spe, p. 145-159, 2016.

FEIGIN, V.L.; NIKITIN, Y.U.; BOTS, M.L.; VINOGRADOVA, T.E.; GROBBEE, D.E. A population-based study of the associations of stroke occurrence with weather parameters in Siberia, Russia (1982-92). European Journal of Neurology, 7(2), 171-178, 2000.

FIRPO, M.Â.F.; SANSIGOLO, C.A.; ASSIS, S.V. Climatologia e variabilidade sazonal do número de ondas de calor e de frio no Rio Grande do Sul associadas ao ENOS. Revista Brasileira de Meteorologia, v.27, n.1, p. 95-106, 2012.

FM/UFMG - Faculdade de Medicina da Universidade Federal de Minas Gerais. Risco de infarto e males circulatórios podem aumentar no frio. Notícias Externas, 2014. Disponível em: <http://site.medicina.ufmg.br/inicial/risco-deinfarto-e-malescirculatorios>. Acesso em: 22 mar. 2018.

GASPARRINI, A.; GUO, Y.; HASHIZUME, M.; LAVIGNE, E.; ZANOBETTI, A.; SCHWARTZ, J.; TOBIAS, A.; TONG, S.; ROCKLÖV, J.; FORSBERG, B.; LEONE, M.; SARIO, M. BELL, M.L.; GUO, Y.L.; WU, C.; KAN, H.; YI, S.; COELHO, M.S.Z.S.; SALDIVA, P.H.N.; HONDA, Y.; KIM, H.; ARMSTRONG, B. Mortality risk attributable to high and low ambient temperature: a multicountry observational study. The Lancet, p. 1-7, 2015.

GGWS - Golden Gate Weather Services. El Niño and La Niña years and intensities: based on Oceanic Niño Index (ONI). 2018. Disponível em: <http://ggweather.com/enso/oni.htm>. Acesso em: 22 mar. 2018.

GRIMM, A.M. Clima da região sul do Brasil. In: Tempo e Clima no Brasil. Iracema F.A. Cavalcanti [et al.] organizadores. São Paulo: Oficina de Textos, 2009, Cap. 17 , p. 259-275.

GRIMM, A.M. Variabilidade interanual do clima no Brasil. In: Tempo e Clima no Brasil. Iracema F.A. Cavalcanti [et al.] organizadores. São Paulo: Oficina de Textos, 2009, Cap. 22, p.353-374.

GUERREIRO, V.I.V. Mortalidade e conforto bioclimático em Coimbra - estudo da vulnerabilidade das populações ao frio. Dissertação (Mestrado em Geografia Humana) - Faculdade de Letras, Universidade de Coimbra. Coimbra, 2011.

HEALY, J.D. Excess winter mortality in Europe: a cross country analysis identifying key risk factors. Journal of Epidemiology and Community Health; 57:784-9, 2003.

IBGE - Instituto Brasileiro de Geografia e Estatística. Censo demográfico 2000 e pesquisa de orçamentos familiares: 2002/2003. 2003. Disponível em: <http://www.ibge.gov.br/estadosat/temas.php?sigla=sc\&tema=mapapobreza20 03>. Acesso em: 28 mai. 2015.

Censo demográfico 2010: cidades@. 2010. Disponível em: <http://www.cidades.ibge.gov.br/xtras/home.php>. Acesso em: 16 abr. 2014.

. Estimativas de população: downloads. 2017. Disponível em: <https://www.ibge.gov.br/estatisticas-novoportal/sociais/populacao/9103estimativas-de-populacao.html?edicao $=9113 \& \mathrm{t}=$ downloads $>$. Acesso em: 22 mar. 2018. 
INMET - Instituto Nacional de Meteorologia. Banco de Dados Meteorológicos para Ensino e Pesquisa - BDMEP. 2018. Disponível em: <http://www.inmet.gov.br/projetos/rede/pesquisa/form_mapas_c_diario.php>. Acesso em: 02 fev. 2018.

KÖPPEN, W.; GEIGER, R. Handbuch der klimatologie. Gebrüder Borntraeger, Berlin, $1936 . \quad$ Disponível em: <https://Das_geographische_System_der_Klimate_\%281936\%29.pdf>. Acesso em: 22 mar. 2018.

MENDONÇA, F.; DANNI-OLIVEIRA, I.M. Climatologia: noções básicas e climas do Brasil. $1^{\text {a }}$ rev. - São Paulo: Oficina de Textos, 206 p., 2007.

MONTEIRO, M.; FURTADO. S.M. O clima do trecho Florianópolis - Porto Alegre: uma abordagem dinâmica. Revista Geosul, nº 19 /20, p. 117-133, 1995.

MONTEIRO, M.A.; MENDONÇA, M. Dinâmica atmosférica do estado de Santa Catarina. In: Atlas de Desastres Naturais do Estado de Santa Catarina: período de 1980 a 2010. HERRMANN, M.L.P. (org), 2. ed. atual. e rev. - Florianópolis: IHGSC/Cadernos Geográficos, 2014, Cap. 2, p. 5-12.

MORABITO, M.; CRISCI, A.; GRIFONI, D.; ORLANDINI, S.; CECCHI, L.; BACCI, L.; MODESTI, P.; GENSINI, G.; MARACCHI, G. Winter air-mass-based synoptic climatological approach and hospital admissions for myocardial infarction in Florence, Italy. Environmental Research, n. 102, p. 52-60, 2006.

MORETTIN, P.A.; BUSSAB, W.O. Estatística básica. $8^{a}$ ed. São Paulo: Saraiva, 2013.

MS - Ministério da Saúde. Manual do Sistema de Informações Hospitalares do SUS (SIH/SUS). Brasília - DF, dez., 2004. Disponível em: <http://sna.saude.gov.br/download/Manual\%20>. Acesso em: 22 mar. 2018.

OMS - Organização Mundial da Saúde. Classificação estatística internacional de doenças e problemas relacionados à saúde - CID-10. OMS, tradução Centro Colaborador da OMS para a Família de Classificações Internacionais em Português, $10^{a}$ rev., V.I, São Paulo: editora da USP, 2008. Disponível em: <http://www.datasus.gov.br/cid10/V2008/WebHelp/i20_i25.htm>. Acesso em: 11 jun. 2015.

PNUD - Programa das Nações Unidas para o Desenvolvimento. Atlas do Desenvolvimento Humano no Brasil: perfil municipal - São Joaquim, SC. 2010. Disponível em: <http://www.atlasbrasil.org.br/2013/pt/perfil_m/saojoaquim_sc>. Acesso em: 03dez. 2015.

RODRIGUES, M.S. Dicionário brasileiro de estatística. Rio de Janeiro, $2^{\mathrm{a}}$ ed.: Instituto Brasileiro de Estatística, 1970, 350 p.

ROGERSON, P.A. Métodos estatísticos para geografia: um guia para o estudante. Porto Alegre, 3a ed.: Bookman, 2012, 348 p.

SCHMITZ, C.M. A precipitação de neve no Brasil meridional. Dissertação (Mestrado em Geografia) - Departamento de Geografia, Universidade Federal do Rio Grande do Sul, Porto Alegre, 2007.

SIH/SUS - Ministério da Saúde - Sistema de Informações Hospitalares do SUS. Informações de Saúde (TABNET): Epidemiológicas e Morbidade. Brasília, 2015. Disponível

em: 
<http://www2.datasus.gov.br/DATASUS/index.php?area=0203>. Acesso em: 10 jun. 2015.

SILVA, S.P. Modelação e previsão do efeito do FRIo Extremo na SAúde da população: a base para o desenvolvimento de um sistema de alerta em tempo real-FRIESA. Dissertação (Mestrado em Bioestatística) - Faculdade de Ciências, Universidade de Lisboa, Lisboa, 2014.

SILVEIRA, R.B.; ALVES, M.P.A.; MENDONÇA, M. O frio como desastre socionatural e a sua relação com doenças vinculadas ao aparelho respiratório no município de Biguaçu e na microbacia São Mateus - Santa Catarina - Brasil. In: Anais do VIII Simpósio Latino-americano de Geografia Física e IV Simpósio Ibero-americano de Geografia Física, Santiago do Chile, p.1218-1226, 2014.

SILVEIRA, R.B.; ROCHA, G.S.; ALVES, M.P.A. Contabilização das ondas de frio em Curitiba - Paraná. In: XVII Simpósio Brasileiro de Geografia Física Aplicada, p. 2162-2172, 2017. Disponível em: <https://ocs.ige.unicamp.br/ojs/sbgfa/article/view/2434>. Acesso em: 03 fev. 2018.

SOUZA, R.O. A ocorrência de neve em planaltos subtropicais: o caso do sul do Brasil. Dissertação (Mestrado em Geografia) - Faculdade de Filosofia, Letras e Ciências Humanas, Universidade de São Paulo, São Paulo, 1997.

TORTORA, G.J. O Sistema Circulatório. In: Corpo humano: fundamentos de anatomia e fisiologia. Porto Alegre: Armed Editora, 2000, 574 p.

VAVRUS, S.; WALSH, J.E.; CHAPMAN, W.L.; PORTIS, D.The behavior of extreme cold air outbreaks under greenhouse warming. International Journal of Climatology, n. 26, p. 1133-1147, 2006.

WHO - World Health Organization. Extreme weather and climate events and public health responses: report on a WHO meeting. Bratislava, Slovakia, 09-10 february, 48 p., 2004.

WILKINSON, P.; LANDON, M.; ARMSTRONG, B.; STEVENSON, S.; McKEE, M. Cold comfort: the social and environmental determinants of excess winter death in England, 1986-1996. York, United Kingdom: Joseph Rowntree Foundation, 2001. 